\title{
Graphene oxide exacerbates dextran sodium sulfate-induced colitis via ROS/AMPK/p53 signaling to mediate apoptosis
}

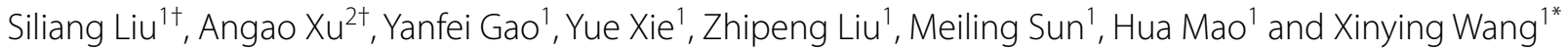

\begin{abstract}
Background: Graphene oxide (GO), a novel carbon-based nanomaterial, has promising applications in biomedicine. However, it induces potential cytotoxic effects on the gastrointestinal (GI) tract cells, and these effects have been largely uncharacterized. The present study aimed to explore the toxic effects of GO on the intestinal tract especially under pre-existing inflammatory conditions, such as inflammatory bowel disease (IBD), and elucidate underlying mechanisms.

Results: Our findings indicated that oral gavage of $\mathrm{GO}$ worsened acute colitis induced by $2.5 \%$ dextran sodium sulfate (DSS) in mice. In vitro, GO exacerbated DSS-induced inflammation and apoptosis in the FHC cell line, an ideal model of intestinal epithelial cells (IECS). Further, the potential mechanism underlying GO aggravated mice colitis and cell inflammation was explored. Our results revealed that GO treatment triggered apoptosis in FHC cells through the activation of reactive oxygen species (ROS)/AMP-activated protein kinase (AMPK)/p53 pathway, as evidenced by the upregulation of cytochrome c (Cytc), Bax, and cleaved caspase-3 (c-cas3) and the downregulation of Bcl-2. Interestingly, pretreatment with an antioxidant, $\mathrm{N}$-acetyl-L-cysteine, and a specific inhibitor of AMPK activation, Compound C (Com.C), effectively inhibited GO-induced apoptosis in FHC cells.

Conclusions: Our data demonstrate that GO-induced IECs apoptosis via ROS/AMPK/p53 pathway activation accounts for the exacerbation of colitis in vivo and aggravation of inflammation in vitro. These findings provide a new insight into the pathogenesis of IBD induced by environmental factors. Furthermore, our findings enhance our understanding of $\mathrm{GO}$ as a potential environmental toxin, which helps delineate the risk of exposure to patients with disturbed intestinal epithelial barrier/inflammatory disorders such as IBD.
\end{abstract}

Keywords: Graphene oxide, Colitis, Intestinal epithelial cell, Apoptosis, Reactive oxygen species, AMP-activated protein kinase

\section{Background}

Inflammatory bowel disease (IBD) is a chronic, non-specific inflammatory condition of the gastrointestinal (GI) tract. The principal types of IBD are ulcerative colitis

\footnotetext{
*Correspondence: helenwxy@smu.edu.cn

† Siliang Liu and Angao Xu co-first author

1 Department of Gastroenterology, Zhujiang Hospital, Southern Medical

University, Guangzhou 510515, People's Republic of China

Full list of author information is available at the end of the article
}

and Crohn's disease [1]. In recent years, the incidence of IBD has gradually increased worldwide [2]. It is well documented that abnormal immunological reactions caused by genetic and environmental factors contribute to the development of IBD [3]. However, the exact underlying mechanisms remain unclear and require further investigation.

With the development of nanoparticle technology, the safety of nanoparticles has garnered substantial attention among researchers. Graphene oxide (GO), a promising 
derivative of graphene, possesses a large surface area and contains many surface functional groups compared with pristine graphene, which renders it as an attractive candidate for drug delivery, bone regeneration, antibiosis and even detection of pathogenic bacteria [4-8]. A previous study reported that the market for GO in 2020 could reach $\$ 618$ million [9]. Along with an increase of GO in production and application, there has been an increase in concern over the unintentional or occupational exposure of GO and its subsequent impact on human health [10]. Until now, multiple studies have provided evidence that GO could be directly cell permeable or readily engulfed via endocytosis into tissues and cells, leading to the induction of adverse effects [11-13]. For instance, inflammation response in the lungs was observed in mice after GO exposure [14-16]. To the best of our knowledge, the toxic effects of GO on cells have been extensively studied and exposure to GO could cause a decrease in cell viability, alterations in the cell cycle, and apoptosis [17-19].

The intestinal tract is regarded as the primary site of interaction with nanomaterials, which renders the uptake of nanoparticles complicated because of the role of the intestinal barrier [20]. Although multiple studies have demonstrated the adverse impacts of GO on human health, only limited information on its effect on GI tract is available. In a systemic in vivo study conducted to detect the biodistribution of GO after intravenous injection and oral gavage, the results obtained suggested that the absorption of oral GO through the intestinal tract is ineffectual [15]. However, limited information still suggested that exposure to graphene family of nanomaterials alters intestinal barrier permeability by inducing apoptosis or changes in gut microbiota [21,22]. Given the above findings, the exact toxic effects of GO on the intestinal tract and the underlying molecular mechanisms have not been systematically elucidated.

Intestinal epithelial cells (IECs) play a key role in maintaining the balance between the immune response and tissue homeostasis, especially as the apoptosis of IECs contributes to the chronic inflammation of the gut, such as during IBD [23]. Previous studies have provided evidence that GO-induced cytotoxicity promotes apoptosis through the activation of various signaling pathways, such as p38 mitogen-activated protein kinase signaling cascade and extracellular signal-regulated kinase signaling pathway $[24,25]$. Adenosine monophosphateactivated protein kinase (AMPK), which is a conserved energy sensor, plays a crucial role in the antioxidant defense of cells and modulates cellular activities such as proliferation, cell cycle progression, and apoptosis [26]. Currently, the effects of GO on IECs apoptosis and the underlying mechanisms, such as the AMPK-related signaling pathway, remain unclear.
In the present study, we explored the potential toxicity of GO on the intestinal tract based on colitis induced by dextran sodium sulfate (DSS) and investigated the mechanism involved in vitro. Our study provides new insight into the pathogenesis of IBD, as it is related to environmental factors, and advances the current understanding of the risk of environmental exposure to GO.

\section{Results}

\section{Characterization of GO}

To characterize GO used in this study, we used different microscopy techniques and observed our GO samples. Representative atomic force microscopy (AFM) images of GO are shown in Fig. 1a. Most GO were found to exist in a single layer or a few layers with a thickness of $\sim 1.0$ $\mathrm{nm}$ and a lateral dimension ranging from 200 to 300 $\mathrm{nm}$ (Fig. 1b), which is consistent with the basic characteristics of GO nanosheets. As depicted in Fig. 1c, GO also showed a monolayer structure with sharp edges via transmission electron microscopy (TEM). The size distribution of GO in water is shown in Fig. 1d. Based on the Raman spectra results, we significantly observed two distinctive $\mathrm{D}$ and $\mathrm{G}$ peaks at $1344 \mathrm{~cm}^{-1}$ and $1602 \mathrm{~cm}^{-1}$, respectively (Fig. 1e). Furthermore, we measured the average hydrodynamic particle sizes and zeta potentials of GO in different media using dynamic light scanning (DLS). As shown in Table 1, at $24 \mathrm{~h}$, the average hydrodynamic particle size in water was $195.7 \pm 0.8 \mathrm{~nm}$, while in phosphate-buffered saline (PBS) and culture medium, it increased to $248.0 \pm 1.4 \mathrm{~nm}$ and $333.5 \pm 0.8 \mathrm{~nm}$, respectively. Further, GO showed the most negative zeta potential in sterile water, followed by in PBS and culture medium, indicating GO was prone to aggregate in culture medium or PBS.

\section{Oral administration of GO nanoparticles aggravated DSS-induced colitis}

To evaluate whether GO exposure affects colitis in vivo, we studied four experimental groups of mice. From days 4 to 6, the weight of mice in the DSS-wild-type (DSS-WT) and DSS-GO groups continuously decreased compared with that of mice in the WT and WT-GO groups. DSSinduced colitis in mice was observed to be a spontaneous limited disease, and the mice have gradually recovered from the weight loss after removing DSS-solution. However, after day 8, the DSS-GO group mice still showed a significant reduction in body weight compared with the DSS-WT group mice (Fig. 2b), and this was accompanied by an obvious shortening of the colon (Fig. 2c, d). In addition, hematoxylin and eosin (H\&E)-stained sections of colonic tissues showed severe inflammatory cells infiltration (Fig. 2e), and the total histological score indicated severe disruption of the mucosal epithelium in the 
a

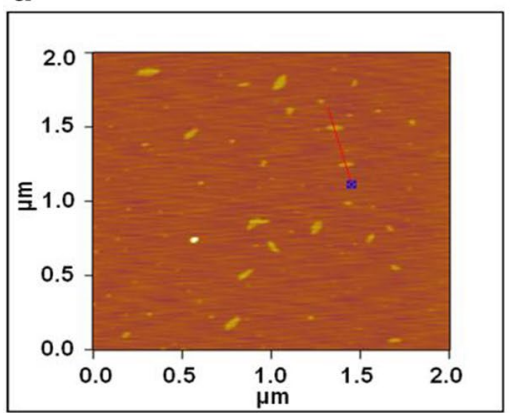

C

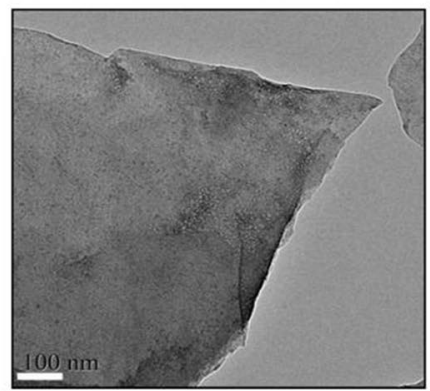

b

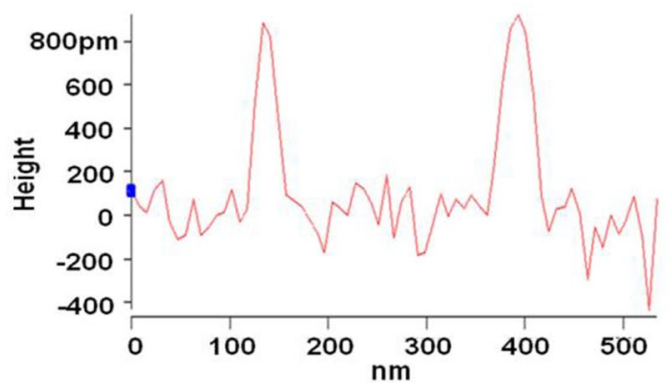

d

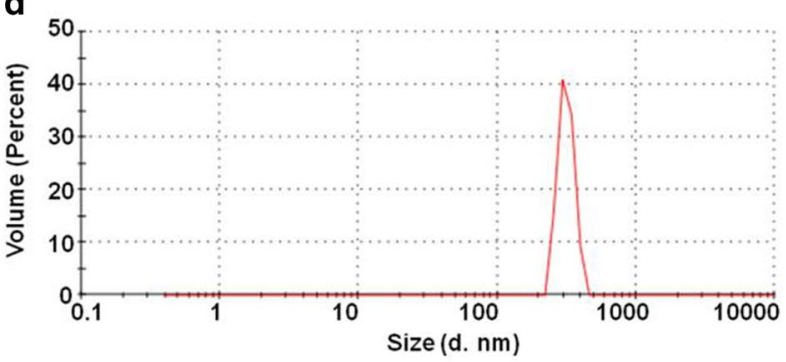

e

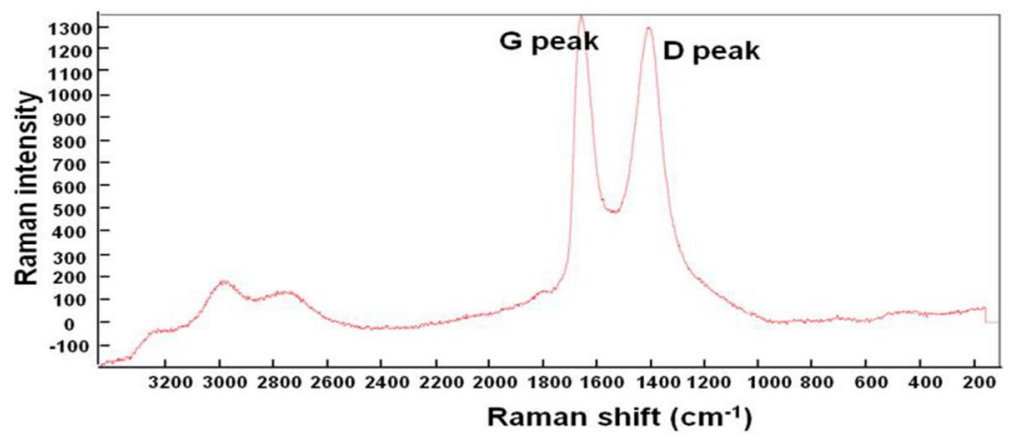

Fig. 1 The characterization of GO. a, b Representative AFM images of GO. c Representative TEM image of GO. Scale bar: $100 \mathrm{~nm}$. $\mathbf{d}$ The size distribution of GO in water using DLS. e Raman spectroscopy of GO nanosheets

Table 1 Stability and dispersity of GO nanoparticles in different media

\begin{tabular}{|c|c|c|c|c|c|c|}
\hline \multirow{2}{*}{$\begin{array}{l}\text { GO } \\
\text { Time }\end{array}$} & \multicolumn{2}{|l|}{ In water } & \multicolumn{2}{|l|}{ In PBS } & \multicolumn{2}{|c|}{ In culture medium } \\
\hline & Size $(n m)$ & Zeta potential (mV) & Size $(n m)$ & Zeta potential (mV) & Size $(n m)$ & Zeta potential (mV) \\
\hline $12 \mathrm{~h}$ & $159.9 \pm 0.8$ & $-14.4 \pm 2.6$ & $95.4 \pm 0.8$ & -14.2 & $146.8 \pm 0.2$ & -7.1 \\
\hline $24 \mathrm{~h}$ & $195.7 \pm 0.8$ & -26.8 & $248.0 \pm 1.4$ & -12.5 & $333.5 \pm 0.8$ & -8.8 \\
\hline $3 d$ & $259.2 \pm 0.7$ & $-16.5 \pm 4.0$ & $255.6 \pm 2.1$ & -17.0 & $321.4 \pm 2.1$ & $-16.4 \pm 0.8$ \\
\hline $5 d$ & $294.8 \pm 0.6$ & -18.7 & $296.4 \pm 0.9$ & $-14.6 \pm 0.9$ & $370.3 \pm 2.4$ & -9.4 \\
\hline $7 d$ & $311.8 \pm 2.2$ & -17.7 & $342.9 \pm 1.2$ & -17.1 & $1271 \pm 3.9$ & -3.4 \\
\hline
\end{tabular}

GO graphene oxide, $P B S$ phosphate-buffered saline

DSS-GO group, compared with DSS-WT group (Fig. 2f). Interestingly, mice that received $\mathrm{GO}$ in the absence of colitis showed no significant differences in weight, colon length, and histological scores from the corresponding parameters of mice in the WT group. 

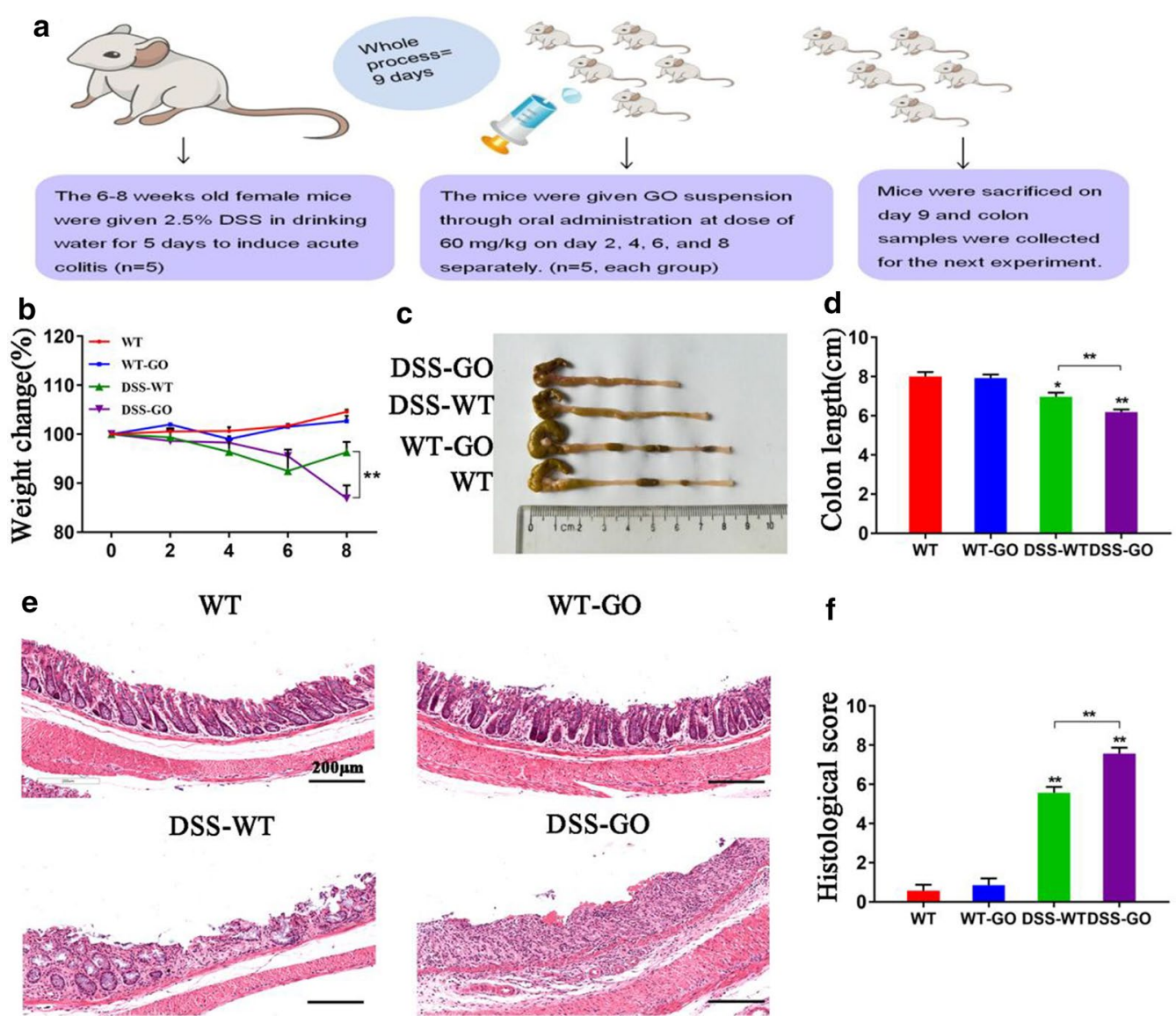

f
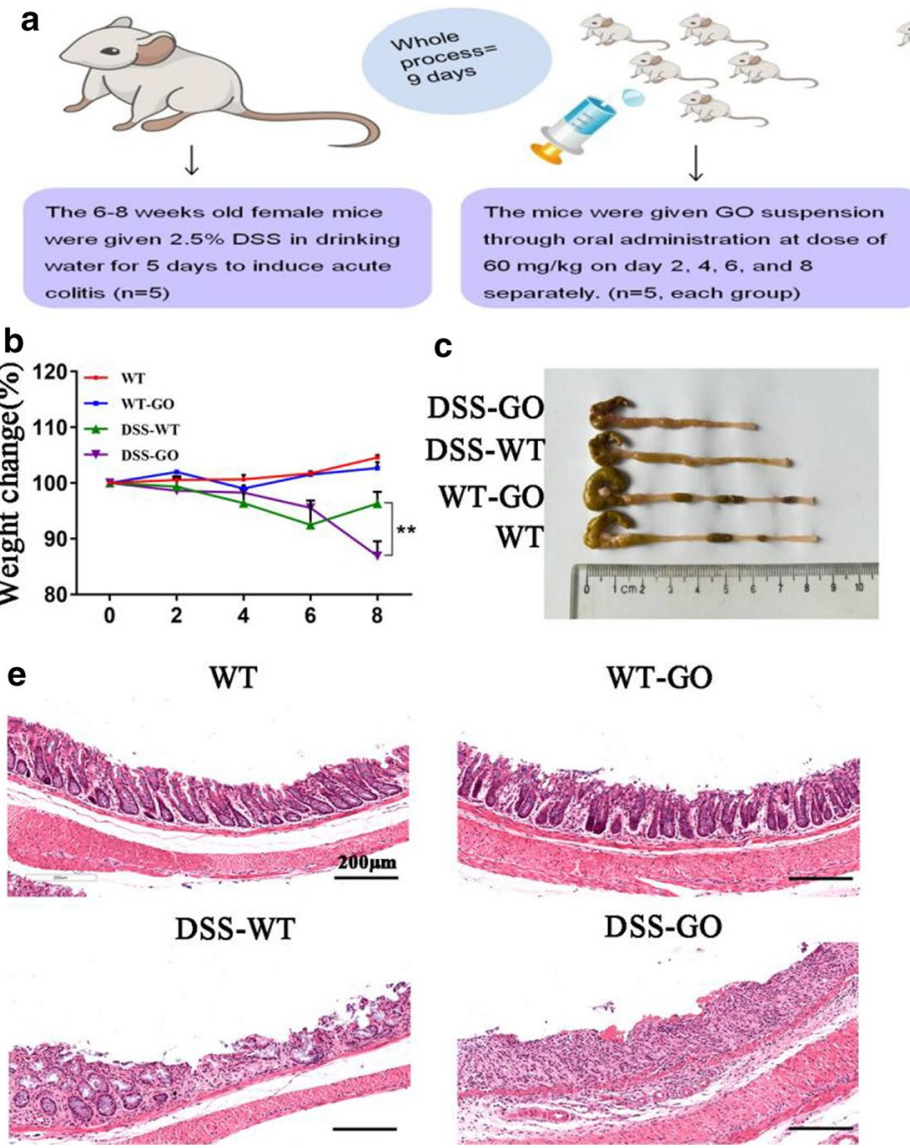

c

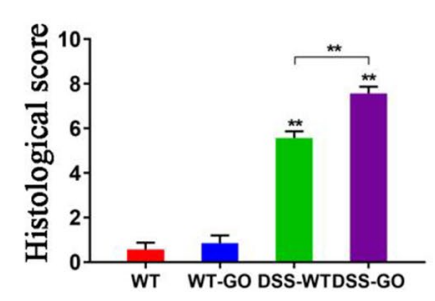

Fig. 2 Administration of GO by oral gavage aggravated DSS-induced colitis in mice. a Schematic illustrations of experiments on the intestinal toxicity of mice after repeated oral exposure to GO. Mice were sacrificed on day 9 to collect colon samples. $\mathbf{b}$ Weight changes and $\mathbf{c}$, $\mathbf{d}$ colon length were measured. Body weight was presented as the percentage of the initial weight (on day 0). e Representative H\&E images of colon tissues. Scale bar: $200 \mu \mathrm{m}$. f The total histological score was calculated as the sum of the score for epithelial damage and the infiltration score. Data are presented as the means \pm SEMs from three independent experiments in all analyses. ${ }^{*} P<0.05,{ }^{*} P<0.01$

To further evaluate the inflammatory response to GO in the intestinal tract, we detected the expression of several important inflammatory cytokines, which play an important role in colitis. Our results showed that GO treatment in the absence of colitis did not cause obvious changes in IL-6, IL-17, and IFN- $\gamma$ expression (Fig. 3a). However, the expression of these pro-inflammatory cytokines increased in the DSS-WT and DSS-GO groups, while IL-10 expression decreased significantly (Fig. 3a). Besides, the DSS-GO group further promoted the release of pro-inflammatory cytokines than DSS-WT group.

For further research on the effects of GO on intestinal inflammation, we finally assessed intestinal apoptosis through terminal deoxynucleotidyl transferase-mediated nick end labeling (TUNEL) staining. Based on Fig. 3b, an obvious green fluorescence in the intestinal epithelium in DSS-GO group could be seen, compared with the DSS-WT group, indicating a higher rate of apoptosis in the former. This may have been the cause of exacerbating colitis but still required further investigation.

\section{The uptake of GO in IECs exacerbated cell inflammation induced by DSS}

Given the aforementioned in vivo results, we next investigated the relationship between GO and IECs inflammation induced by DSS in vitro. First, we evaluated the biological activity of FHC cells, an ideal in vitro model of IECs, following GO treatment. As depicted in Fig. 4a, we found that GO was taken up into FHC cells and mainly distributed in the cytoplasm via TEM observation. Additionally, when compared with the control group and single fluorescein isothiocyanate (FITC)-bovine serum albumin (BSA) group, the FITC-BSA-labeled GO was detected on the cytoplasm of FHC cells after $24 \mathrm{~h}$ of incubation, as green-colored FITC-fluorescence signals, as shown in Fig. 4b. Meanwhile, a small amount of FITCfluorescence was also detected in the cytomembrane. 

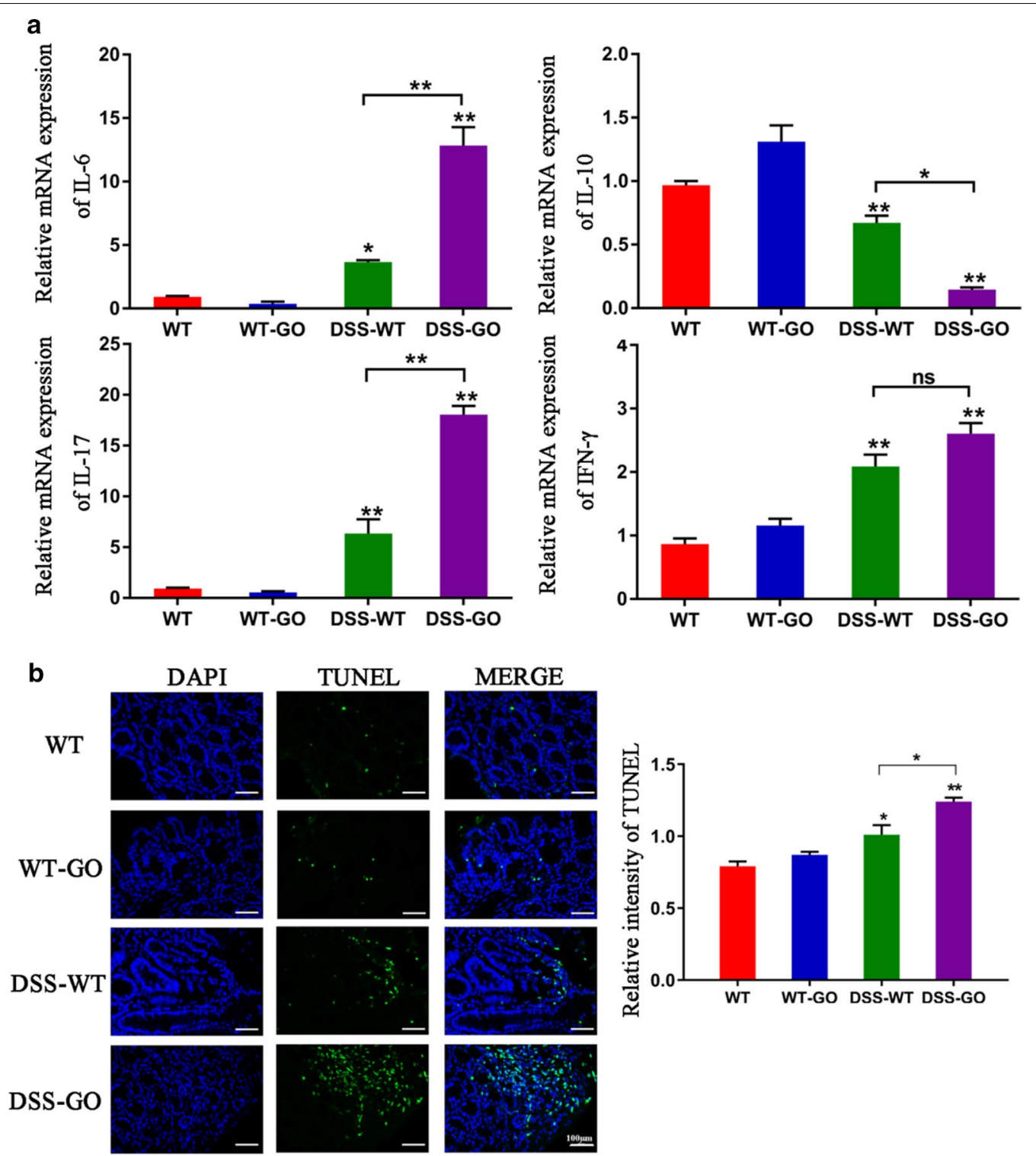

Fig. $3 \mathrm{GO}$ induced the production of pro-inflammatory cytokines and apoptosis in mice with DSS-induced colitis. a The relative mRNA expression of IL-6, IL-10, IL-17, and IFN- $\gamma$ in mice was detected through qPCR. b Apoptosis in the intestinal tract was detected using TUNEL assay. Scale bar: 100 $\mu \mathrm{m} .{ }^{*} P<0.05,{ }^{*} P<0.01$

In addition, the results of flow cytometric analysis also showed that the fluorescence density of the FITC-BSAGO group was significantly increased, compared to that in the FITC-BSA and control group (Fig. 4c), which further confirmed the absorption of GO by FHC cells. Subsequently, the potential toxic effects of GO on cell viability were detected. We found that $\mathrm{GO}$ significantly decreased FHC cell viability in a dose- and time-dependent manner (Fig. 4d). GO treatment at a concentration of $50 \mu \mathrm{g} / \mathrm{mL}$ for $24 \mathrm{~h}$ resulted in approximately $50 \%$ cell death compared with the cell death rate of the control group.

Next, the cell membrane integrity was evaluated using lactic dehydrogenase (LDH) release. It is seen from Fig. 4e that the LDH release from FHC cells significantly increased from almost 0 (control group) to more than $50 \%$ upon $50 \mu \mathrm{g} / \mathrm{mL}$ GO treatment. In addition, $\mathrm{LDH}$ release increased with a longer duration of GO exposure, which corresponds with the cell viability results. Based on the cell viability and LDH release results, we chose an 

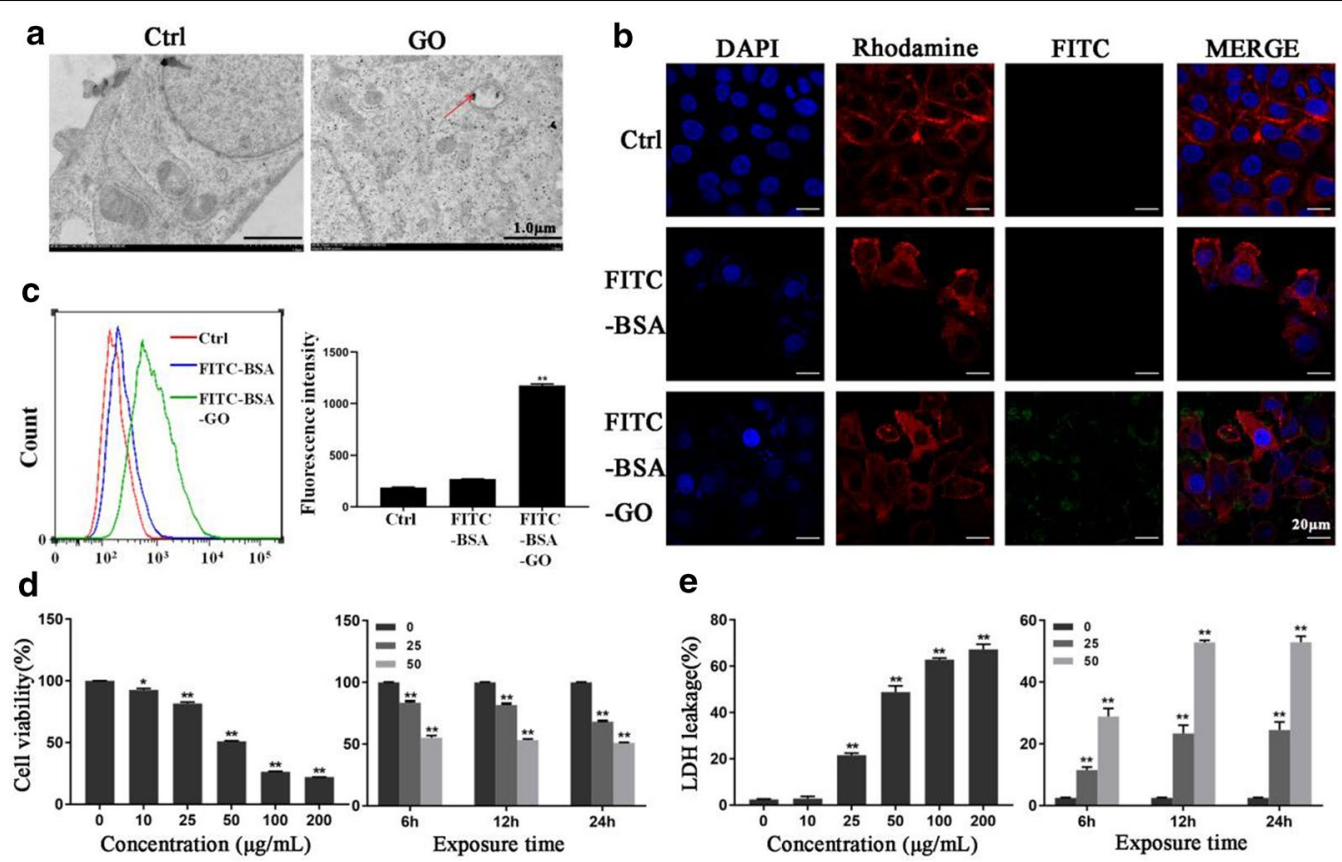

Fig. 4 The uptake of GO in IECs induced dose- and time-dependent toxicity based on cell viability and LDH release. a The uptake of GO in FHC cells following $50 \mu \mathrm{g} / \mathrm{mL}$ GO treatment for $24 \mathrm{~h}$. The red arrows indicate GO accumulation in the cytoplasm under TEM observation. Scale bar: $1 \mu \mathrm{m}$. b, c GO was labeled by FITC-BSA and exposed to FHC cells for additional $24 \mathrm{~h}$. Untreated cells were served as control group, while single FITC-BSA exposed to FHC cells was included as negative control group. The green fluorescence of FITC-BSA labeled GO on the cytoplasm and cytomembrane of FHC cells using confocal microscopy. Scale bar: $20 \mu \mathrm{m}$. The relative fluorescence intensity was measured using flow cytometry. $\mathbf{d}$, e Relative cell viability and LDH release in cultured FHC cells incubated with $\mathrm{GO}$ at different concentrations $(0,10,25,50,100$, or $200 \mu \mathrm{g} / \mathrm{mL})$ for $24 \mathrm{~h}$, or $25 \mathrm{and} 50$ $\mu \mathrm{g} / \mathrm{mL}$ GO for 6, 12, and $24 \mathrm{~h}$. The results were expressed as means \pm SEMs from three independent experiments. ${ }^{*} P<0.05,{ }^{*} P<0.01$

appropriate concentration of GO (25 and $50 \mu \mathrm{g} / \mathrm{mL})$ for subsequent experiments.

For the purpose of verifying the potential effects of $\mathrm{GO}$ on intestinal inflammation in vitro, FHC cells were administered 1\% DSS treatment prior to GO treatment to induce inflammation model in vitro. As shown in Fig. 5a, GO induced significant cell death (>45\%) compared to that in the control group after a 24-h exposure, whereas GO treatment in combination with DSS resulted in $58 \%$ cell death. Next, we investigated the inflammatory responses of FHC cells exposed to GO. As shown in Fig. 5b, GO and DSS both stimulated the release of IL-17, IFN- $\gamma$ and TNF- $\alpha$. Moreover, obvious inflammatory responses were observed in the DSS + GO group compared to that in the GO group, as evidenced by increased mRNA expression of IL-17 and IFN- $\gamma$. In addition, intracellular reactive oxygen species (ROS) production after GO treatment in the presence or absence of DSS was also tested. It was seen from the results of Fig. $5 \mathrm{c}$ that intracellular ROS production increased significantly after GO and DSS treatment. Moreover, GO further stimulated ROS generation with DSS treatment. Finally, we tested the effects of GO on apoptosis in FHC cells with or without DSS treatment. The results obtained showed that the percentage of apoptotic cells in FHC cells increased from $7.09 \%$ (ctrl) to $17.2 \%$ (GO group) and $12.02 \%$ (DSS group), which is shown in Fig. 5d. However, the percentage of apoptotic cells in the DSS $+\mathrm{GO}$ group reached $24.9 \%$. These results are in line with in vivo results.

\section{$\mathrm{GO}$ induced mitochondrial dysfunction, ROS production and apoptosis in FHC cells}

To explore the potential mechanism underlying the aggravation of colitis and cell inflammation mediated by $\mathrm{GO}$, we further investigated the toxic responses to GO in FHC cells, focusing on cell apoptosis and the expression of related proteins. Firstly, we explored the mitochondrial structure and function following GO exposure. Apparent mitochondrial swelling and rupture were observed by TEM after GO exposure (Fig. 6a). Next, we tested the mitochondrial membrane potential (MMP), an important manifestation of early apoptosis, to evaluate mitochondrial function after GO exposure. Following GO treatment, we observed a GO-induced decrease of MMP (Fig. 6b, c). Moreover, intracellular ROS generation was detected. As shown in Fig. 6d, e, GO enhanced the levels of intracellular ROS. Cell apoptosis was also evaluated through flow cytometry. As shown in Fig. 6f, the 

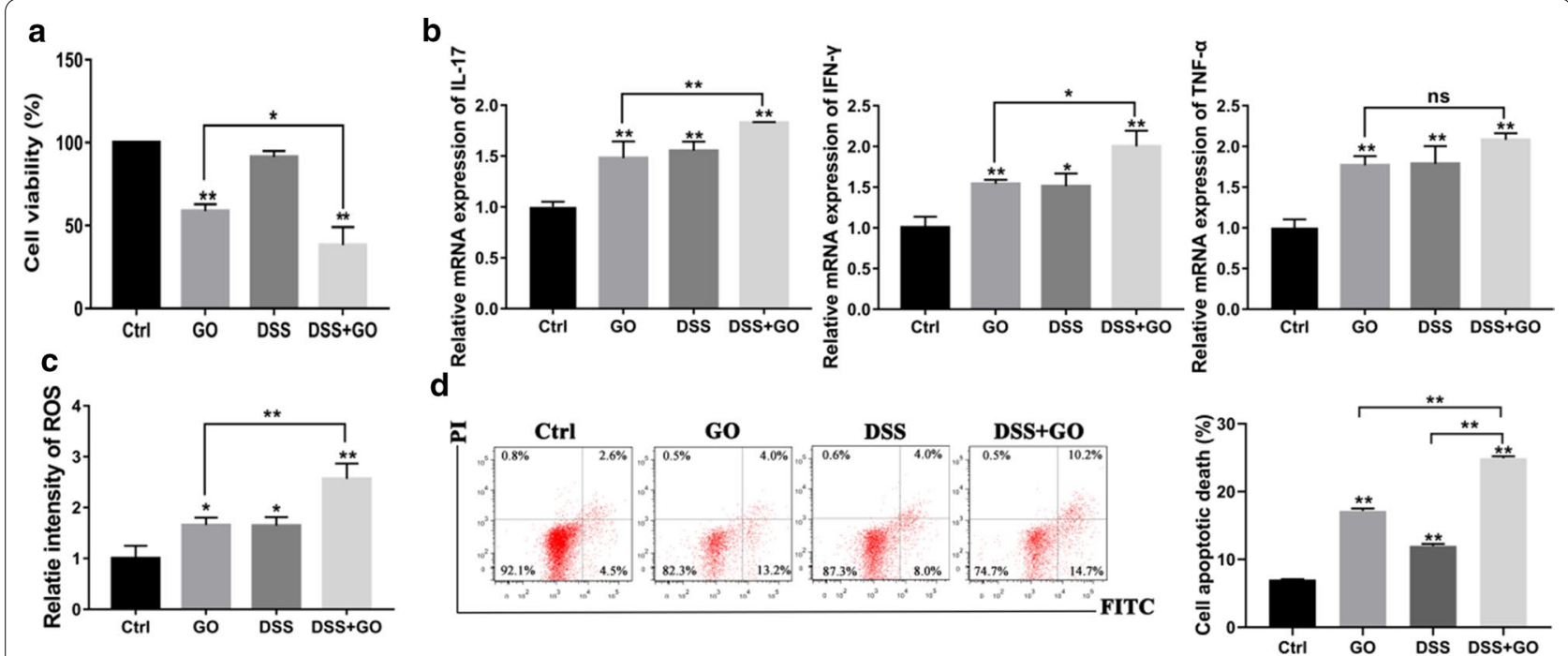

Fig. 5 GO further exacerbated the cell inflammation induced by DSS in IECS. FHC cells were treated with $1 \%$ DSS for 0 or $4 \mathrm{~h}$ prior to culture in the presence or absence of GO. a CCK-8 was performed to assess the cell viability. $\mathbf{b}$ The relative mRNA expression of IL-17, IFN- $\gamma$ and TNF-a using qPCR. c The intracellular production of ROS was tested using flow cytometry. $\mathbf{d}$ Flow cytometry was carried out to measure cell apoptosis. Apoptotic cell death was expressed as the sum of the percentage of early apoptotic cells and late apoptotic cells. The results were expressed as means \pm SEMs from three independent experiments. ${ }^{*} P<0.05,{ }^{* *} P<0.01$

percentages of apoptotic cells were $25.32 \%$ in the control group, 39.5\% among FHC cells exposed to $25 \mu \mathrm{g} / \mathrm{mL} \mathrm{GO}$, an approximately $50 \%$ among FHC cells exposed to 50 $\mu \mathrm{g} / \mathrm{mL}$ GO.

To reveal the potential mechanism responsible for cell apoptosis upon GO treatment, we detected the apoptosis-related proteins, with a focus on cytochrome c (Cytc), Bax, Bcl-2 and cleaved caspase-3 (c-cas3). As shown in Fig. $6 \mathrm{~g}$ using western blot, GO treatment resulted in increased expression of Cytc, Bax, c-cas3 and decreased expression of Bcl-2, consistent with the induction of apoptosis.

Taken together, our results demonstrated that GO induced apoptosis, as evidenced by mitochondrial damage, Cytc release and ROS overproduction. To investigate the association between Cytc and ROS, we used minocycline (MC, an inhibitor of Cytc) before GO treatment and found that expressions of Cytc and intracellular ROS production were all reduced (Fig. 6h, i), which implied that GO-induced ROS generation resulted from mitochondrial dysfunction.

\section{GO-induced apoptosis was regulated via ROS generation through the AMPK/p53 pathway in FHC cells}

Given that the AMPK pathway plays an important role in cellular apoptosis, we investigated the effect of GO treatment on the expression of proteins involved in the AMPK pathway, including AMPK, PI3K, AKT, and p53. The levels of phosphorylated AMPK and p53 were significantly increased after GO treatment for $24 \mathrm{~h}$ (Fig. 7a, b), whereas the levels of phosphorylated PI3K and AKT were not (Fig. 7c, d).

To further examine the relationship among GOinduced apoptosis, ROS overproduction, and AMPK/p53 activation, we treated FHC cells with $\mathrm{N}$-acetyl-L-cysteine (NAC), an ROS scavenger, and compound C (Com.C), a specific AMPK inhibitor, before incubation with GO. As shown in Fig. 8a, b, NAC significantly abrogated the GO-induced intracellular ROS accumulation. Additionally, flow cytometry showed significant reduction in GO-induced apoptosis after treatment of FHC cells with NAC and Com.C, from $58 \%$ to $29.6 \%$ and $28.3 \%$, respectively (Fig. 8c). Moreover, pre-treating the FHC cells with NAC and Com.C resulted in the downregulated expression of c-cas3 and Bax and increased the protein expression of Bcl-2 (Fig. 8d, e). Finally, the western blot analysis showed that treatment with NAC and Com.C inhibited the phosophorylation of AMPK $\alpha$ (Thr172) and p53 in FHC cells (Fig. 8f, g). These results suggested that GOinduced apoptosis was regulated via the ROS/AMPK/p53 signaling pathway.

\section{Discussion}

Herein, we aimed to find whether GO, a common environmental toxin, could negatively impact individuals with a defective intestinal barrier such as patients with IBD. We estimated the potential toxic effects of GO on a DSS-induced colitis mouse model and revealed the 


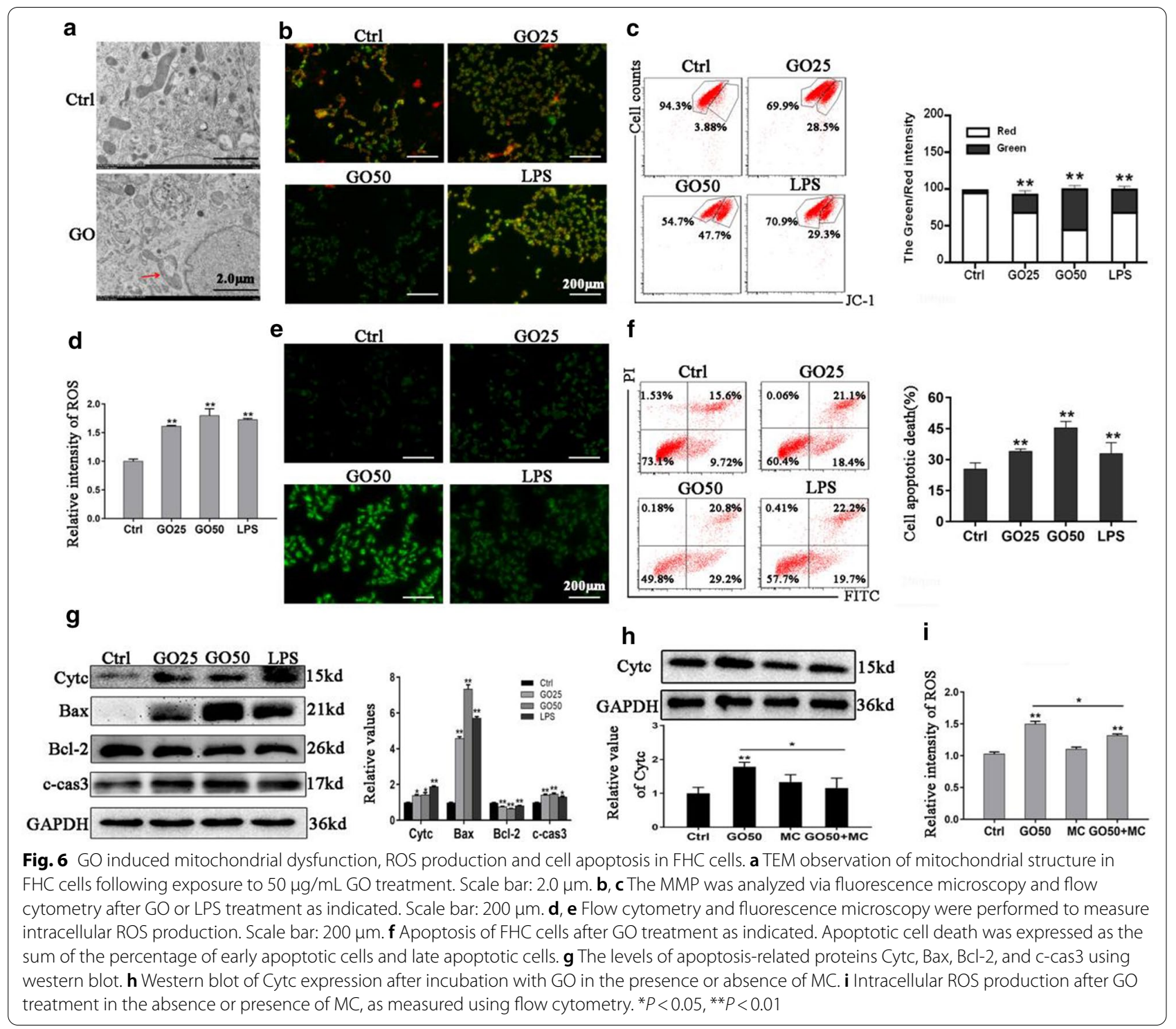

potential mechanisms in vitro. GO, a promising nanomaterial with a wide range of applications in biomedicine, has currently garnered tremendous research interest. Its extensive application means that the issue of potential GO toxicity worsens with the increasing environmental exposure. The intestinal tract is usually regarded as the primarily targeted organ by nanomaterials due to its direct exposure to the external environment. Nevertheless, the current understanding of the mechanism behind GO toxicity on the intestinal tract is poorly understood. Only a few in vivo studies were performed to address the hazard of GO following oral exposure [27-29]. Damage to the intestinal mucosal barrier is a defining characteristic of chronic intestinal inflammatory disorders such as IBD [30]. Based on the present findings, we proposed that there may be a close association between GO and chronic intestinal inflammation, which has not been studied/reported adequately. IBD has become a global health issue with its incidence continually increasing particularly in countries undergoing increasing westernization, thus underscoring the vital role of environmental factors [31].

In particular, the physiochemical properties of nanomaterials including the surface charge, lateral size, thickness, and aggregation status play an important role in the interactions with cells, organs, and tissues [32]. It is extremely critical for us to acquire detailed information on GO before evaluating its safety. For this purpose, we first characterized the GO used in this study. The results demonstrated that the commercial GO purchased from 


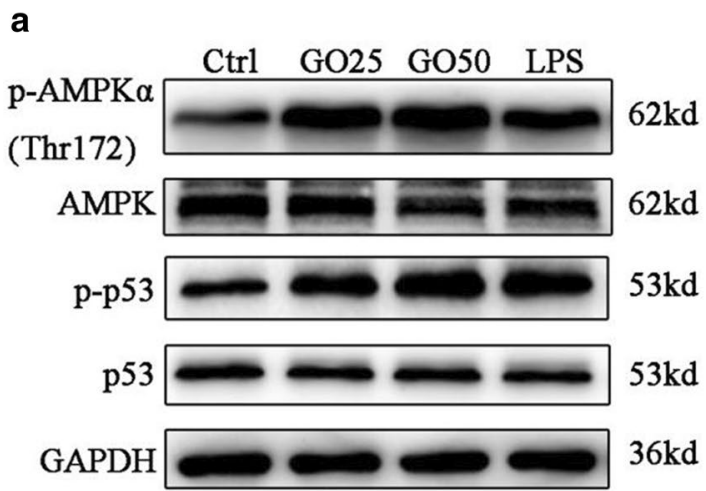

C
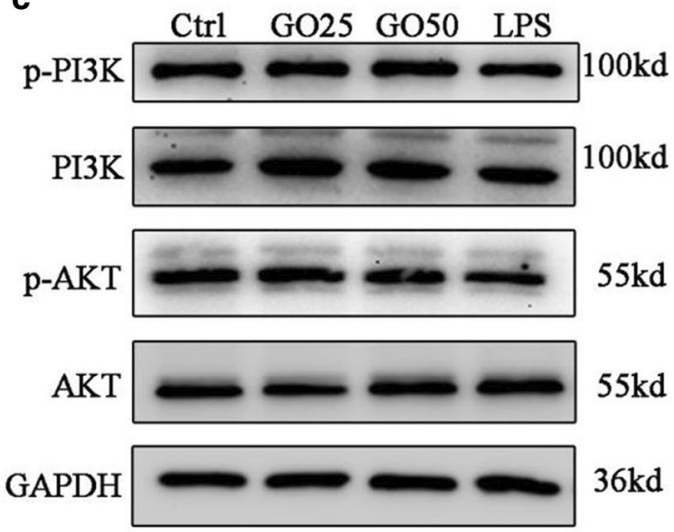

b

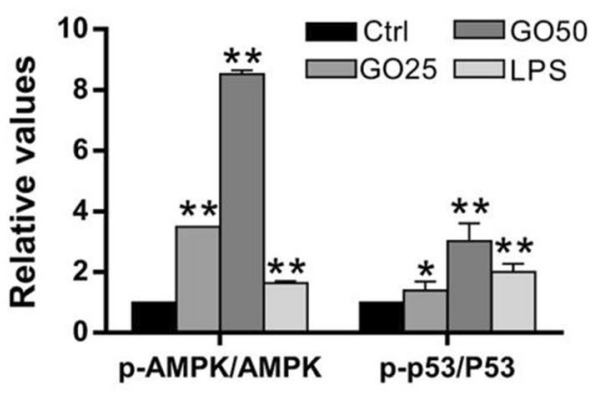

d

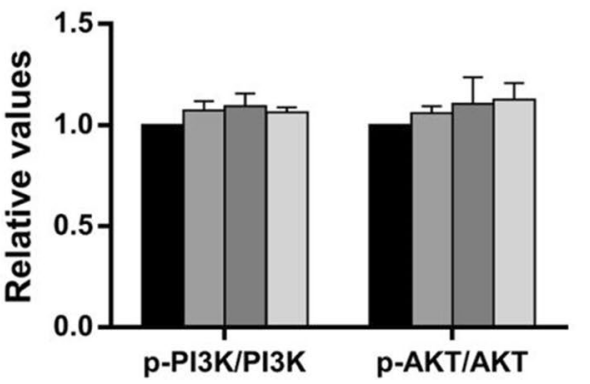

Fig. 7 GO activated the AMPK/p53 signaling pathway in FHC cells. FHC cells were treated with 0, 25, or 50 $\mu \mathrm{g} / \mathrm{mL}$ GO for $24 \mathrm{~h}$, and LPS treated cells served as the positive control group. a-d Cell lysates were subjected to western blot to analyze the expression of p-AMPKa (Thr172), AMPK, p-p53, p53, p-PI3K, PI3K, p-AKT, and AKT. Changes in the levels of the phosphorylated proteins were quantified after normalization against the corresponding pan proteins. ${ }^{*} P<0.05,{ }^{* *} P<0.01$

Sigma had a single to a few layers with sizes ranging from 200 to $300 \mathrm{~nm}$. However, the hydrodynamic particle sizes of GO in PBS and culture medium gradually increased over time than those in water. This indicated that GO was prone to aggregation owing to the highly salted environment and the presence of protein components, which is consistent with the results of previous studies [33, 34].

Next, in vivo and in vitro experiments were carried out to assess the biosafety of GO. The in vivo results revealed that acute oral administration of GO increased the extent of colitis, accompanied by the release of pro-inflammatory cytokines (IL-6, IL-17, and IFN- $\gamma$ ) and apparent apoptosis in the intestinal mucosal epithelium. However, GO-treated mice in the absence of colitis did not show any inflammation in the intestinal tract compared with the mice in the control group, which is in line with a previous study [15]. It is noteworthy that other nanoparticles, including titanium dioxide and silica nanoparticle, have also been shown to increase intestinal inflammatory response [27, 35, 36]. Therefore, we hypothesized that the absorption of nanoparticles is enhanced significantly in the state of inflammation with a defective intestinal barrier, resulting in a further inflammatory response. These results suggest that GO is harmful in the presence of preexisting inflammatory disorders, such as IBD. Furthermore, in vitro experiments were conducted to explore the potential mechanism for the role of GO in colitis.

In the present study, we focused on the direct toxic effects of GO on IECs, which play an important role in the development of chronic inflammation including IBD. Currently, most in vitro studies have chosen to use a human colon adenocarcinoma cell (Caco-2) line as a model to explore the interaction of GO with IECs [37, 38]. However, evidence indicated that the uptake of GO is closely related to the differentiation status of Caco-2 [39]. Therefore, in our study we chose the FHC cell line, which is also commonly used as an in vitro model of IECs, to explore the potential mechanism responsible for exacerbating colitis. Our data showed that the uptake of GO resulted in dose- and time-related alterations of cell viability, which correlates with the results of other studies in different cell models $[17,40,41]$. Considering that GO 
a

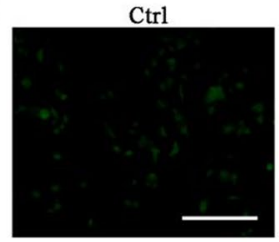

C

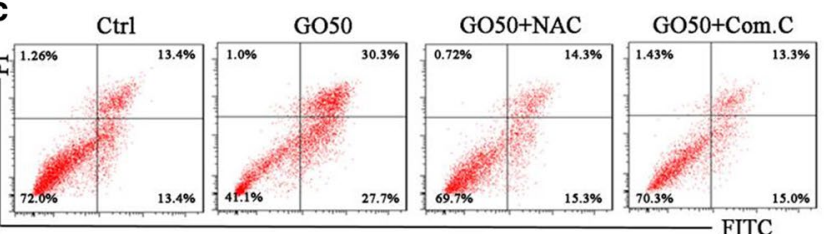

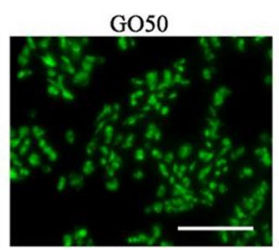

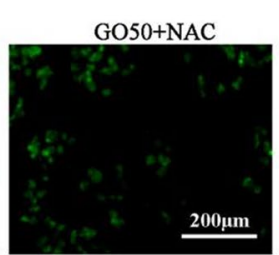

e
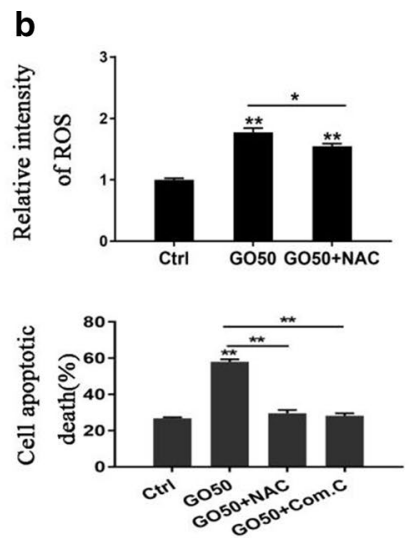

d

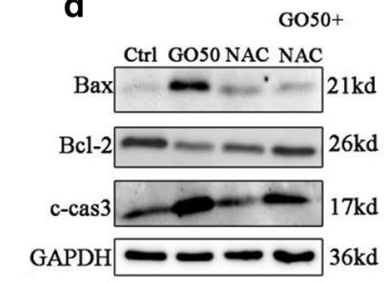

f

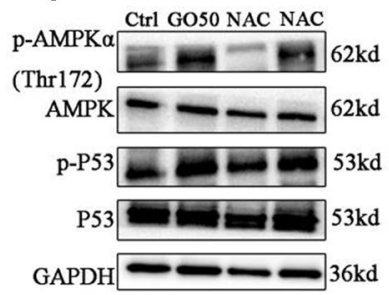

$\cos x$

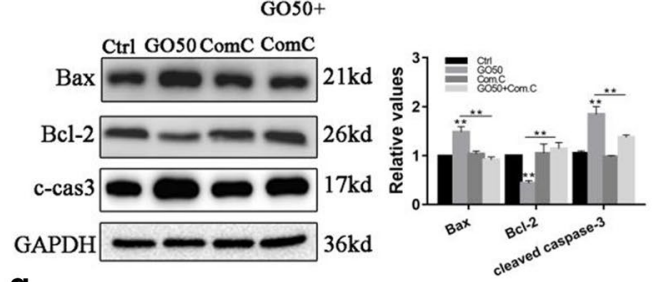

g

GO50+

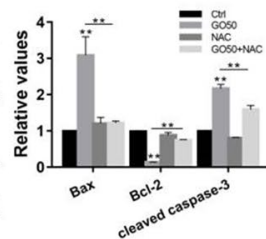

-AMPK Ctrl GO50ComC ComC
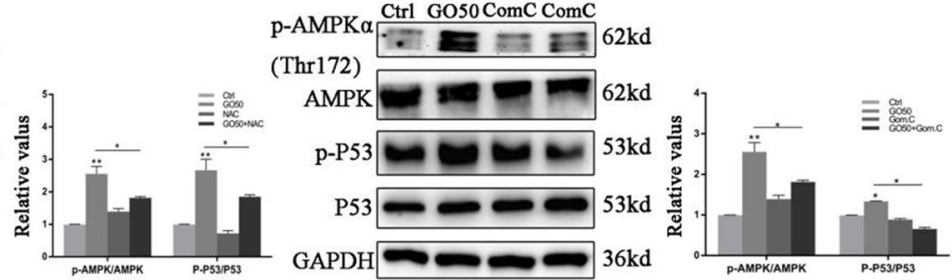

Fig. 8 GO-induced apoptosis in FHC cells was regulated via the ROS/AMPK/p53 pathway. Cells were incubated with $50 \mu \mathrm{g} / \mathrm{mL} \mathrm{GO}$ in the absence or presence of $400 \mu \mathrm{M}$ NAC or $10 \mu \mathrm{M}$ Com.C. $\mathbf{a}, \mathbf{b}$ The intracellular ROS level was measured using DCFHDA detection through fluorescence microscopy and flow cytometry. Scale bar: $200 \mu \mathrm{m}$. c Flow cytometry was used to evaluate the apoptotic rate of FHC cells after exposure to GO. d, e The relative expression of Bax, Bcl-2, and c-caspase 3 was measured using western blot. $\mathbf{f}, \mathbf{g}$ The relative expression of AMPK, p-AMPK, p53 and p-p53 was measured following GO treatment in the absence or presence of NAC and Com.C. Changes in the levels of these proteins were quantified after normalization against GAPDH or AMPK and p53. ${ }^{*} P<0.05,{ }^{*} P<0.01$

was also detected on the cytoplasmembrane of FHC cells using confocal microscopy, we also explored the effect of GO on cell membrane integrity and found obvious LDH release from FHC cells following GO treatment. Further, we found that GO exacerbated cell inflammation induced by DSS in FHC cells, as evidenced by decreased cell viability, elevated expression of IL-17 and IFN- $\gamma$, increased ROS production, and obvious apoptosis compared to those with GO treatment, which are consistent with the results of in vivo experiments. Furthermore, we explored the mechanism responsible for the aggravating effects of GO on colitis and cell inflammation. It is commonly accepted that the inflammatory response, DNA damage, apoptosis, oxidative stress, necrosis, and autophagy are involved in the toxicological mechanisms of GO [25, 42, 43]. Generally, intracellular ROS generation, reflecting the oxidative stress status, induces lipid peroxidation, protein inactivation, mitochondrial dysfunction, eventually leading to apoptosis [44]. Based on our data, the destruction of mitochondrial structure, decreased MMP, and increased level of intracellular ROS were observed in GO-treated IECs. We also observed notable apoptosis in IECs following GO exposure.

To the best of our knowledge, several apoptotic signals, such as DNA damage and cell stress as well as nanomaterial exposure, contribute to the activation of the $\mathrm{Bcl}-2$ family of proteins and promote permeabilization of the mitochondrial membrane, further resulting in the release of pro-apoptotic proteins, such as Cytc and Bax. As a result of the formation of the apoptosome, caspase- 9 and caspase- 3 are activated, which then triggers apoptosis [45-47]. In our study, the GO-induced apoptosis is followed by the loss of MMP and the increasing in intracellular ROS level, which indicated that the mitochondrial 
pathway may be primarily responsible for this effect. To investigate this, we performed western blot, which showed that GO exposure caused increased Cytc, Bax, and cleaved-caspase 3 expression, but decreased Bcl-2 expression. It has been suggested that Cytc is an essential molecular component of the electron transport chain, which promotes ROS generation [48]. In the present study, the inhibition of Cytc significantly prevented the intracellular ROS production induced by GO, which implied that GO-induced intracellular ROS accumulation resulted from mitochondrial dysfunction. Furthermore, prior studies have demonstrated that various signaling pathways are activated by ROS and numerous related proteins, such as MAPK, JNK and AMPK, are involved in the apoptotic process [49-51]. Notably, AMPK plays an important role in the regulation of apoptosis through modulating its downstream signaling molecules including p53, JNK, and mTOR [52-55]. To characterize the AMPK/p53 pathway involved, we measured the levels of AMPK and p53, as well as their phosphorylated forms. We found that GO activated the AMPK/p53 pathway. Furthermore, we found that Com.C treatment led to a significant decrease in apoptosis, which confirmed the crucial role of the AMPK/p53 pathway in GO-induced apoptosis. Cytosolic p53 translocates to the mitochondrial surface and directly interacts with Bcl-2 family of proteins, further leading to the release of Bax and the activation of apoptosis [56]. We also observed an increased expression of $\mathrm{Bcl}-2$ and reduced expression of Bax after pretreatment with Com.C. Moreover, the fact that NAC effectively inhibited the activation of AMPK suggested that ROS was an upstream molecule of AMPK activation, which is consistent with a previous study [57].

As mentioned above, GO exposure to DSS-treated mice resulted in severe intestinal inflammation. Exposure of IECs to GO induced the loss of MMP and the generation of intracellular ROS. Subsequently, ROS activated the AMPK/p53 pathway to trigger apoptosis. This could be the main mechanism underlying the exacerbation of colitis by GO. However, concerning the underlying mechanism responsible for GO exacerbation of colitis, our study only focused on ROS release and IECs apoptosis. There might be other mechanisms of apoptosis that could be involved, and these need further investigation.

\section{Conclusions}

In summary, our findings demonstrate that the oral administration of GO exacerbates DSS-induced acute colitis via the activation of the ROS/AMPK/p53 signaling pathway to mediate apoptosis in IECs. Our study expands the understanding of GO toxicity in the GI tract and provides new insight into the biocompatibility of graphene materials, indicating that further research is needed. In addition, our findings suggest that individuals with a preexisting intestinal inflammatory condition, such as IBD, must be cautious when unintentionally exposed to GO or its derivatives.

\section{Materials and methods \\ GO characterization}

Commercially produced GO powder, purchased from Sigma-Aldrich (USA), was dispersed in pure water to prepare a stock solution $(1 \mathrm{mg} / \mathrm{mL})$. Before characterization and subsequent experiments, the stock solution was sonicated for $2 \mathrm{~h}(40 \mathrm{kHz}$, power 99\%) using an ultrasonic processor (Biosafer, China). For characterization, the prepared GO sample was separately placed on mica and copper plates with 200 mesh grids for assessment via AFM (Bruker, USA) and TEM (Hitachi, USA). The structure of GO was assessed using Raman spectroscopy (Renishaw, UK) with a 514-nm laser. To acquire detailed information on its physicochemical properties, GO was dissolved in pure water, $\mathrm{PBS}$, and complete culture medium for $12 \mathrm{~h}, 24 \mathrm{~h}, 3$ days, 5 days, and 7 days. Then, the average hydrodynamic particle size and zeta potential were analyzed using DLS (Malvern, UK). For DLS measurements, $1.2 \mathrm{~mL}$ of GO sample solution in the cuvette accepted the light from the laser and the process of each sample was conducted at least 12 runs. The photodiode detector (Malvern, UK) was used to acquire the DLS signals and then processed with Zetasizer nanoapplication software (Malvern, UK).

\section{Animal experimentation}

Female C57BL/6 mice (6-8 weeks old and weighing 18-20 g), purchased from the Animal Research Center of Southern Medical University (Guangzhou, China), were housed in a specific pathogen-free facility. The mice were divided into four groups ( $\mathrm{n}=5$ per group): WT mice without DSS or GO treatment (WT group); WT mice treated with GO (WT-GO group); WT mice treated with DSS to induce colitis (DSS-WT group); and mice with DSS-induced colitis exposed to GO (DSSGO group). To generate the acute colitis model, female C57BL/6 mice were administered 2.5\% DSS (MP, USA) orally with drinking water for 5 days and then received normal drinking water for 3 days. Mice were exposed to GO separately via oral gavage at a dose of $60 \mathrm{mg} / \mathrm{kg} /$ day on days $2,4,6$, and 8 . During the process, the mice were monitored daily to observe for weight change, diarrhea, and rectal bleeding. A schematic representation of the animal protocol is provided in Fig. 2 a. 


\section{H\&E and TUNEL staining assay}

Mice were sacrificed on day 9, and colon samples were collected and fixed in $4 \%$ paraformaldehyde, sectioned, and stained with $H \& E$ for examination using light microscopy. Histological scoring was conducted following a previously described system [58]. For the TUNEL assay, colon slices were stained with Reagent 1 (TdT) and Reagent 2 (dUTP) at a mass ratio of 2:29 following the instruction of the TUNEL kit for $1 \mathrm{~h}$ before nuclear staining with 4',6-diamidino-2-phenylindole dihydrochloride (DAPI, Beyotime, China). After washing with PBS thrice, fluorescence microscopy (Nikon, Japan) was conducted to observe the apoptotic cells in the intestinal tract and collect images (UV excitation wavelength 330-380 nm, emission wavelength $420 \mathrm{~nm}$; FITC green light excitation wavelength 465-495 nm, emission wavelength 515-555 $\mathrm{nm})$.

\section{Cell culture}

A human colon epithelium cell line, the FHC cell line, purchased from the American Type Culture Collection (ATCC, VA) was used as an ideal in vitro model of IECs because it could form a confluent layer, thus allowing us explore the cell membrane integrity [59]. FHC cell line was maintained in RPMI-1640 supplemented with 10\% fetal bovine serum (FBS), $100 \mathrm{U} / \mathrm{mL}$ penicillin, and 100 $\mathrm{mg} / \mathrm{mL}$ streptomycin (Gibco, USA). FHC cells were cultured in a humidified atmosphere at $37{ }^{\circ} \mathrm{C}$ with $5 \% \mathrm{CO}_{2}$.

\section{Cell viability and membrane integrity assay}

Cell counting kit (CCK)-8 (Dojingdo, Japan) and LDH assays were performed to evaluate the cell viability and cell membrane integrity, respectively. FHC cells were seeded in 96-well plates at a density of $5 \times 10^{3}$ cells/per well and incubated overnight for adherence. GO was introduced into cells at various concentrations $(10,25$, 50,100 , and $200 \mu \mathrm{g} / \mathrm{mL}$ ) for $24 \mathrm{~h}$ or incubated with 25 and $50 \mu \mathrm{g} / \mathrm{mL}$ GO for 6,12 , and $24 \mathrm{~h}$ to investigate the cytotoxic effect of $\mathrm{GO}$ on FHC cells in concentration and time aspects, untreated cells served as the control group. For DSS-stimulated inflammation in vitro, FHCs were administered 1\% DSS dissolved in RPMI-1640 for $0 \mathrm{~h}$ (ctrl group) or $4 \mathrm{~h}$ (DSS group) prior to co-incubation with GO (GO and DSS + GO group), which was reported previously [59]. For the CCK-8 assay, at the end of treatment, the culture medium was removed and the cells were washed with PBS three times. Precisely 10 $\mu \mathrm{L}$ of CCK-8 working buffer was added to each well and incubated for an additional $1 \mathrm{~h}$ at $37^{\circ} \mathrm{C}$. The optical density of each well at $450 \mathrm{~nm}$ was read using a microplate reader (Molecular Device, USA). For the LDH assay, after $24 \mathrm{~h}$ of co-incubation with GO as indicated above, the 96-well plate was centrifuged (400 g, $5 \mathrm{~min}$ ) and $60 \mu \mathrm{L}$ of supernatant from each well was separately transferred to another 96-well plate following the manufacturer's instructions. The optical density of each well was read using a microplate reader at $490 \mathrm{~nm}$.

\section{Detection of inflammatory cytokines}

Colon samples were collected for the detection of inflammatory cytokines. In vitro, $500 \mu \mathrm{L}$ FHC cells were seeded on 12 -well plates at a density of $5 \times 10^{4}$ cells/well and incubated overnight. Then, cells were pretreated with $1 \%$ DSS for $0 \mathrm{~h}$ or $4 \mathrm{~h}$, followed by $50 \mu \mathrm{g} / \mathrm{mL}$ GO treatment for another $24 \mathrm{~h}$. Total RNA from colon tissues and cells were extracted using Trizol reagent (Gibco, USA) and quantified using the NanoDrop spectrophotometer (Thermo Fisher, USA). Quantitative real-time PCR (qPCR) was conducted and analyzed using LightCycler 480 (Roche, Switzerland). The inflammatory cytokine primers used are listed in Table 2.

\section{TEM observations of GO uptake and mitochondrial structure}

Exactly $1 \times 10^{6} \mathrm{FHC}$ cells were cultured in 6-well plates and exposed to $\mathrm{GO}$ as indicated. The cells were collected via centrifuging after $24 \mathrm{~h}$ of incubation and fixed with $3 \%$ glutaraldehyde, post-fixed in osmium tetroxide, dehydrated in ethanol, and then polymerized using epoxy resin. GO uptake as well as the intracellular

Table 2 The primers of inflammatory cytokines used in the study

\begin{tabular}{|c|c|c|}
\hline Gene name & Organism & Primer sequence \\
\hline \multirow[t]{2}{*}{ GAPDH } & \multirow[t]{2}{*}{ Mus musculus } & GGGTCCCAGCTTAGGTTCAT \\
\hline & & TACGGCCAAATCCGTTCACA \\
\hline \multirow[t]{2}{*}{$\mid \mathrm{L}-6$} & \multirow[t]{2}{*}{ Mus musculus } & TTCACAAGTCGGAGGCTTA \\
\hline & & CAAGTGCATCATCGTTGTTC \\
\hline \multirow[t]{2}{*}{ IL-10 } & \multirow[t]{2}{*}{ Mus musculus } & GGAAGAGAAACCAGGGAGA \\
\hline & & CCACAGTTTTCAGGGATGA \\
\hline \multirow[t]{2}{*}{ IL-17 } & \multirow[t]{2}{*}{ Mus musculus } & TTCACTTTCAGGGTCGAGA \\
\hline & & GGGGTTTCTTAGGGGTCA \\
\hline \multirow[t]{2}{*}{ IFN- $\gamma$} & \multirow[t]{2}{*}{ Mus musculus } & ACTGGCAAAAGGATGGTG \\
\hline & & GTTGCTGATGGCCTGATT \\
\hline \multirow[t]{2}{*}{ GAPDH } & \multirow[t]{2}{*}{ Homo sapiens } & СCTTCCGTGTCCCCACT \\
\hline & & GCCTGCTTCACCACCTTC \\
\hline \multirow[t]{2}{*}{ IFN- $\gamma$} & \multirow[t]{2}{*}{ Homo sapiens } & CCGCTACATCTGAATGACCTG \\
\hline & & TGGCTTTTCAGCTCTGCATC \\
\hline \multirow[t]{2}{*}{ TNF-a } & \multirow[t]{2}{*}{ Homo sapiens } & ССТCTCTCTAATCAGCCCTCTG \\
\hline & & GAGGACCTGGGAGTAGATGAG \\
\hline \multirow[t]{2}{*}{ IL-17 } & \multirow[t]{2}{*}{ Homo sapiens } & GGATGTTCAGGTTGACCATCAC \\
\hline & & TCCCACGAAATCCAGGATGC \\
\hline
\end{tabular}


mitochondrial structure were observed via high-resolution ht7700 TEM (Hitachi, Japan).

\section{Confocal microscopy and flow cytometry of GO uptake}

According to a previously established method [60], the prepared GO suspension was mixed with FITC-BSA (FITC/BSA $=5: 1$, Bioss Inc., China) at a mass ratio of 1:1 and incubated overnight at $37{ }^{\circ} \mathrm{C}$ in the dark. The mixture was centrifuged at $12,000 \times g$ for $30 \mathrm{~min}$ at $4{ }^{\circ} \mathrm{C}$ and washed briefly with PBS. Then, the pellet was resuspended in the culture medium and added to FHC cultures, which were seeded on sterile coverslips inside culture dishes. Twenty-four hours later, the cells were washed with PBS and fixed with $4 \%$ paraformaldehyde, followed by $0.1 \%$ Triton X-100 permeabilization. Finally, prior to nuclear staining with DAPI, cells were incubated with rhodamine phalloidin (100 $\mathrm{nM})$ for cytoskeleton staining. After washed thrice with PBS, cells were observed under an FV1000 confocal laser scanning microscope (Olympus, Japan). FV10-ASW 3.0 Viewer software was used to analyze the acquired images.

Flow cytometry (BD Biosciences, USA) was also conducted to detect the uptake of GO by FHC cells. $1 \times 10^{6}$ FHC cells were cultured in 6-well plates and then were exposed to FITC-BSA or FITC-BSA-GO, untreated cells served as control group. After $24 \mathrm{~h}$ of treatment, cells were collected and washed by PBS for 2 times, and then these samples were analyzed by flow cytometry immediately.

\section{Cell apoptosis assay}

FHC cells were cultured in 6 -well plates $\left(1 \times 10^{6}\right.$ cells per well). To assess the potential toxic responses to GO with respect to cell inflammation, FHC cells were treated with $1 \%$ DSS for 0 or $4 \mathrm{~h}$ to stimulate inflammation at the cellular level, followed by co-incubation with or without GO $(50 \mu \mathrm{g} / \mathrm{mL})$ for an additional $24 \mathrm{~h}$. To test the toxic effects of GO on IECs, FHC cells were incubated in the presence or absence of either $\mathrm{GO}(0,25$, and $50 \mu \mathrm{g} / \mathrm{mL})$ or $10 \mu \mathrm{g} /$ $\mathrm{mL}$ lipopolysaccharide (LPS, Sigma-Aldrich, USA) for 24 $\mathrm{h}$. To further explore the potential mechanism in vitro, FHC cells were treated with $400 \mu \mathrm{M}$ NAC (MCE, USA), or $10 \mu \mathrm{M}$ Com.C (MCE, USA) for $1 \mathrm{~h}$ before GO incubation $(0$ or $50 \mu \mathrm{g} / \mathrm{mL})$. After incubation as indicated, cells were harvested and washed with PBS three times followed by centrifugation ( $3000 \mathrm{rpm}, 5 \mathrm{~min}$ ). The cell pellet was suspended in $400 \mu \mathrm{L}$ of binding buffer to achieve a density of $1 \times 10^{6}$ cells $/ \mathrm{mL}$. The sample solution was then incubated with $5 \mu \mathrm{L}$ Annexin V-FITC (Beyotime, China) for $15 \mathrm{~min}$ in the dark followed by an additional incubation with $10 \mu \mathrm{L}$ propidium iodide (PI, Beyotime, China) for $5 \mathrm{~min}$. Apoptotic cells were then detected using flow cytometry.

\section{MMP measurement}

Briefly, cells were seeded in a 12-well plate at a density of $5 \times 10^{4}$ cells/well and treated with GO at concentrations of 0,25 , and $50 \mu \mathrm{g} / \mathrm{mL}$, and the cells treated with $10 \mu \mathrm{g} / \mathrm{mL}$ LPS were included in the positive control group. After exposure to GO or LPS, cells were incubated with JC-1 buffer mixture solution (Beyotime, China) for $20 \mathrm{~min}$ at $37{ }^{\circ} \mathrm{C}$ according to the manufacturer's instructions. Fluorescence microscopy and flow cytometry were used to measure the ratio of green (JC-1 monomer)/red (JC-1 aggregates) fluorescence. The increased ration reflected the decrease of MMP.

\section{ROS generation assay}

Intracellular ROS production was measured using the DCFHDA assay kit (Beyotime, China). Specifically, to explore the effect of GO on ROS generation under conditions of DSS-induced inflammation in vitro, $1 \mathrm{~mL}$ of FHC cells (at a density of $5 \times 10^{4}$ cells $/ \mathrm{mL}$ ) was cultured in 12-well plates, incubated with $1 \%$ DSS for 0 or $4 \mathrm{~h}$, and then exposed to $\mathrm{GO}$ treatment at $50 \mu \mathrm{g} / \mathrm{mL}$. To detail the potential mechanisms, $1 \mathrm{~mL}$ FHC cells at density of $5 \times 10^{4}$ cells $/ \mathrm{mL}$ seeded in 12 -well plates were separately treated with LPS and GO $(0,25$, and $50 \mu \mathrm{g} / \mathrm{mL}$ ) or treated with $100 \mu \mathrm{M} \mathrm{MC}$ (Selleck, USA) and NAC before incubaction with GO $(50 \mu \mathrm{g} / \mathrm{mL})$. At the end of treatment, FHC cells were harvested by centrifugation and stained with DCFHDA for $30 \mathrm{~min}$ in the dark at $37{ }^{\circ} \mathrm{C}$. The fluorescence intensity was analyzed using fluorescence microscopy and flow cytometry.

\section{Western blot analysis}

A total of $30 \mu \mathrm{g}$ of protein was separated using $10 \%$ SDS-PAGE and transferred to a PVDF membrane, which was then blocked with 5\% w/v BSA. Membranes were probed with the indicated primary antibodies including rabbit polyclonal antibodies against Cytc, c-cas3, Bcl-2, phosphorylated (p)-AMPK $\alpha$ (Thr172), p-PI3K, PI3K, p-AKT, AKT, p-p53, and p53 (Cell Signaling Technology, USA) along with mouse monoclonal antibodies against Bax and AMPK (Proteintech, China), and GAPDH was used to normalize protein expression. Appropriate secondary antibodies conjugated to horseradish-peroxidase (HRP) were then added and incubated for $1 \mathrm{~h}$. The antigen-antibody complex was detected using an enhanced chemiluminescence reagent (Millipore, USA). The gray intensity of the bands on the western blots was analyzed using the ImageJ software (NIH, Bethesda, USA). 


\section{Statistical analysis}

The experimental data were presented as the mean \pm standard error of the mean (SEM). Differences among the data for the different groups were analyzed using one-way ANOVA. P-values less than 0.05 and 0.01 were considered significant, as indicated.

\begin{abstract}
Abbreviations
AMPK: Adenosine monophosphate-activated protein kinase; AFM: Atomic force microscopy; BSA: Bovine serum albumin; C-cas3: Cleaved caspase-3; CCK: Cell counting kit; Com.C: Compound C; Cytc: Cytochrome c; DAPI: 4',6-Diamidino-2-phenylindole dihydrochloride; DLS: Dynamic light scanning; DSS: Dextran sodium sulfate; FITC: Fluorescein isothiocyanate; Gl: Gastrointestinal; GO: Graphene oxide; HE: Hematoxylin and eosin; IBD: Inflammatory bowel disease; IECs: Intestinal epithelial cells; LDH: Lactic dehydrogenase; LPS: Lipopolysaccharide; MC: Minocycline; MMP: Mitochondrial membrane potential; NAC: N-acetyl-L-cysteine; PBS: Phosphate-buffered saline; PI: Propidium iodide; qPCR: Quantitative real-time PCR; ROS: Reactive oxygen species; SEM: Standard error of the mean; TEM: Transmission electron microscopy; TUNEL: Terminal deoxynucleotidyl transferase-mediated nick end labeling.
\end{abstract}

\section{Acknowledgements}

The authors thank the Guangdong Provincial Key Laboratory of Gastroenterology, Department of Gastroenterology, Nanfang Hospital, Southern Medical University, Guangzhou, Guangdong, China. Moreover, we would like to thank Editage for their writing support on this manuscript.

\section{Authors' contributions}

$S L, A X$, and $Y G$ : animal and cell experiments, drafting the manuscript, data acquisition, data analysis and manuscript revision. YX, ZL and MS: material and technological support, data arrangement and analysis. HM: study concept, and supervision; XW: manuscript revision, study concept, design, supervision, and funding. All authors read and approved the final manuscript.

\section{Funding}

This work was supported by the National Natural Science Foundation of China (Grant Number 81572938).

\section{Availability of data and materials}

The datasets used or analyzed during the current study are available from the corresponding author upon reasonable request.

\section{Declarations}

\section{Ethics approval and consent to participate}

The experimental protocols were approved by the Southern Medical University Ethics and Experimentation of Committee (Grant Number L2016189).

\section{Consent for publication}

All authors agree to be published.

\section{Competing interests}

The authors declare that they have no competing interests.

\section{Author details}

${ }^{1}$ Department of Gastroenterology, Zhujiang Hospital, Southern Medical University, Guangzhou 510515, People's Republic of China. ${ }^{2}$ Huizhou Medicine Institute, Huizhou 516003, People's Republic of China.

Received: 27 June 2020 Accepted: 14 March 2021

Published online: 25 March 2021

\section{References}

1. Baumgart DC, Sandborn WJ. Inflammatory bowel disease: clinical aspects and established and evolving therapies. Lancet. 2007;369:1641-57.
2. Malekzadeh MM, Vahedi H, Gohari K, Mehdipour P, Sepanlou SG, Ebrahimi Daryani $\mathrm{N}$, et al. Emerging epidemic of inflammatory bowel disease in a middle income country: a nation-wide study from Iran. Arch Iran Med. 2016;19:2-15.

3. Lakatos L, Mester G, Erdelyi Z, Balogh M, Szipocs I, Kamaras G, et al. Striking elevation in incidence and prevalence of inflammatory bowel disease in a province of western Hungary between 1977-2001. World J Gastroenterol. 2004;10:404-9.

4. Sun X, Liu Z, Welsher K, Robinson JT, Goodwin A, Zaric S, et al. Nanographene oxide for cellular imaging and drug delivery. Nano Res. 2008;1:203-12.

5. Hussien NA, Isiklan N, Turk M. Pectin-conjugated magnetic graphene oxide nanohybrid as a novel drug carrier for paclitaxel delivery. Artif Cells Nanomed Biotechnol. 2018;46:264-73.

6. Hussein $\mathrm{KH}$, Abdelhamid HN, Zou X, Woo HM. Ultrasonicated graphene oxide enhances bone and skin wound regeneration. Mater Sci Eng C Mater Biol Appl. 2019;94:484-92.

7. Abdelhamid HN, Wu HF. Multifunctional graphene magnetic nanosheet decorated with chitosan for highly sensitive detection of pathogenic bacteria. J Mater Chem B. 2013:1:3950-61.

8. Shahnawaz KM, Abdelhamid HN, Wu HF. Near infrared (NIR) laser mediated surface activation of graphene oxide nanoflakes for efficient antibacterial, antifungal and wound healing treatment. Colloids Surf B Biointerfaces. 2015;127:281-91.

9. Ahmed F, Rodrigues DF. Investigation of acute effects of graphene oxide on wastewater microbial community: a case study. J Hazard Mater. 2013:256-257:33-9.

10. Pelin M, Sosa S, Prato M, Tubaro A. Occupational exposure to graphene based nanomaterials: risk assessment. Nanoscale. 2018:10:15894-903.

11. Li J, Zhang $X$, Jiang J, Wang $Y$, Jiang H, Zhang J, et al. Systematic assessment of the toxicity and potential mechanism of graphene derivatives in vitro and in vivo. Toxicol Sci. 2019;167:269-81.

12. Kersting D, Fasbender S, Pilch R, Kurth J, Franken A, Ludescher M, et al. From in vitro to ex vivo: subcellular localization and uptake of graphene quantum dots into solid tumors. Nanotechnology. 2019;30:395101.

13. Krajnak K, Waugh S, Stefaniak A, Schwegler-Berry D, Roach K, Barger M, et al. Exposure to graphene nanoparticles induces changes in measures of vascular/renal function in a load and form-dependent manner in mice. J Toxicol Environ Health A. 2019;82:711-26.

14. Duch MC, Budinger GRS, Liang YT, Soberanes S, Urich D, Chiarella SE, et al. Minimizing oxidation and stable nanoscale dispersion improves the biocompatibility of graphene in the lung. Nano Lett. 2011;11:5201-7.

15. Yang K, Gong H, Shi X, Wan J, Zhang Y, Liu Z. In vivo biodistribution and toxicology of functionalized nano-graphene oxide in mice after oral and intraperitoneal administration. Biomaterials. 2013;34:2787-95.

16. Park EJ, Lee SJ, Lee K, Choi YC, Lee BS, Lee GH, et al. Pulmonary persistence of graphene nanoplatelets may disturb physiological and immunological homeostasis. J Appl Toxicol. 2017;37:296-309.

17. Feng X, Chen L, Guo W, Zhang Y, Lai X, Shao L, et al. Graphene oxide induces p62/SQSTM-dependent apoptosis through the impairment of autophagic flux and lysosomal dysfunction in PC12 cells. Acta Biomater. 2018;81:278-92.

18. Arbo MD, Altknecht LF, Cattani S, Braga WV, Peruzzi CP, Cestonaro LV, et al. In vitro cardiotoxicity evaluation of graphene oxide. Mutat Res. 2019;841:8-13.

19. Gurunathan S, Arsalan IM, Qasim M, Park CH, Yoo H, Hwang JH, et al. Evaluation of graphene oxide induced cellular toxicity and transcriptome analysis in human embryonic kidney cells. Nanomaterials. 2019;9:969.

20. Neves AR, Queiroz JF, Costa Lima SA, Figueiredo F, Fernandes R, Reis S. Cellular uptake and transcytosis of lipid-based nanoparticles across the intestinal barrier: relevance for oral drug delivery. J Colloid Interface Sci. 2016:463:258-65.

21. Wu Q, Yin L, Li X, Tang M, Zhang T, Wang D. Contributions of altered permeability of intestinal barrier and defecation behavior to toxicity formation from graphene oxide in nematode Caenorhabditis elegans. Nanoscale. 2013;5:9934-43.

22. Chen $H$, Zhao R, Wang B, Zheng L, Ouyang $H$, Wang H, et al. Acute oral administration of single-walled carbon nanotubes increases intestinal permeability and inflammatory responses: association with the changes in gut microbiota in mice. Adv Healthc Mater. 2018;7:e1701313. 
23. Neurath MF, Travis SPL. Mucosal healing in inflammatory bowel diseases: a systematic review. Gut. 2012;61:1619-35.

24. Zhao Y, Zhi L, Wu Q, Yu Y, Sun Q, Wang D. P38 MAPK-SKN-1/Nrf signaling cascade is required for intestinal barrier against graphene oxide toxicity in Caenorhabditis elegans. Nanotoxicology. 2016;10:1469-79.

25. Kang Y, Liu J, Wu J, Yin Q, Liang H, Chen A, et al. Graphene oxide and reduced graphene oxide induced neural pheochromocytoma-derived PC12 cell lines apoptosis and cell cycle alterations via the ERK signaling pathways. Int J Nanomed. 2017;12:5501-10.

26. Afrin MR, Arumugam S, Rahman MA, Karuppagounder V, Sreedhar $\mathrm{R}$, Harima M, et al. Le Carbone, a charcoal supplement, modulates DSS-induced acute colitis in mice through activation of AMPKa and downregulation of STAT3 and caspase 3 dependent apoptotic pathways. Int Immunopharmacol. 2017;43:70-8.

27. Fu C, Liu T, Li L, Liu H, Liang Q, Meng X. Effects of graphene oxide on the development of offspring mice in lactation period. Biomaterials. 2015;40:23-31.

28. Zhang D, Zhang Z, Liu Y, Chu M, Yang C, Li W, et al. The short- and longterm effects of orally administered high-dose reduced graphene oxide nanosheets on mouse behaviors. Biomaterials. 2015;68:100-13.

29. Patlolla AK, Rondalph J, Tchounwou PB. Biochemical and histopathological evaluation of graphene oxide in sprague-dawley rats. Austin J Environ Toxicol. 2017;3:1021.

30. Shen L, Turner JR. Role of epithelial cells in initiation and propagation of intestinal inflammation. Eliminating the static: tight junction dynamics exposed. Am J Physiol Gastrointest Liver Physiol. 2006;290:G577-82.

31. Kaplan GG, Ng SC. Understanding and preventing the global increase of inflammatory bowel disease. Gastroenterology. 2017;152:313.e2-321.e2.

32. Ou L, Song B, Liang H, Liu J, Feng X, Deng B, et al. Toxicity of graphenefamily nanoparticles: a general review of the origins and mechanisms. Part Fibre Toxicol. 2016;13:57.

33. Liu Y, Wang $X$, Wang J, Nie Y, Du H, Dai H, et al. Graphene oxide attenuates the cytotoxicity and mutagenicity of PCB 52 via activation of genuine autophagy. Environ Sci Technol. 2016;50:3154-64.

34. Liao KH, Lin YS, Macosko CW, Haynes CL. Cytotoxicity of graphene oxide and graphene in human erythrocytes and skin fibroblasts. ACS Appl Mater Interfaces. 2011;3:2607-15.

35. Ruiz PA, Moron B, Becker HM, Lang S, Atrott K, Spalinger MR, et al. Titanium dioxide nanoparticles exacerbate DSS-induced colitis: role of the NLRP3 inflammasome. Gut. 2017;66:1216-24.

36. Mu W, Wang Y, Huang C, Fu Y, Li J, Wang H, et al. Effect of long-term intake of dietary titanium dioxide nanoparticles on intestine inflammation in mice. J Agric Food Chem. 2019;67:9382-9.

37. Kucki M, Rupper P, Sarrieu C, Melucci M, Treossi E, Schwarz A, et al. Interaction of graphene-related materials with human intestinal cells: an in vitro approach. Nanoscale. 2016;8:8749-60.

38. Guarnieri D, Sanchez-Moreno P, Del Rio Castillo AE, Bonaccorso F, Gatto F, Bardi G, et al. Biotransformation and biological interaction of graphene and graphene oxide during simulated oral ingestion. Small. 2018;14:e1800227.

39. Kucki M, Diener L, Bohmer N, Hirsch C, Krug HF, Palermo V, et al. Uptake of label-free graphene oxide by Caco-2 cells is dependent on the cell differentiation status. J Nanobiotechnol. 2017;15:46.

40. Frontiñán-Rubio J, Gómez MV, Martín C, González-Domínguez JM, Durán-Prado M, Vázquez E. Differential effects of graphene materials on the metabolism and function of human skin cells. Nanoscale. 2018;10:11604-15.

41. Wei M, Fu Z, Wang C, Zheng W, Li S, Le W. Graphene oxide nanocolloids induce autophagy-lysosome dysfunction in mouse embryonic stem cells. J Biomed Nanotechnol. 2019;15:340-51.

42. Vranic S, Rodrigues AF, Buggio M, Newman L, White MRH, Spiller DG, et al. Live Imaging of label-free graphene oxide reveals critical factors causing oxidative-stress-mediated cellular responses. ACS Nano. 2018;12:1373-89.
43. Gurunathan S, Kang MH, Jeyaraj M, Kim JH. Differential cytotoxicity of different sizes of graphene oxide nanoparticles in leydig (TM3) and sertoli (TM4) cells. Nanomaterials. 2019;9:139.

44. Thubagere A, Reinhard BM. Nanoparticle-induced apoptosis propagates through hydrogen-peroxide-mediated bystander killing: insights from a human intestinal epithelium in vitro model. ACS Nano. 2010;4:3611-22.

45. Cheng WC, Leach KM, Hardwick JM. Mitochondrial death pathways in yeast and mammalian cells. Biochim Biophys Acta. 2008;1783:1272-9.

46. Brenner D, Mak TW. Mitochondrial cell death effectors. Curr Opin Cell Biol. 2009;21:871-7.

47. Brunelle JK, Letai A. Control of mitochondrial apoptosis by the BCl-2 family. J Cell Sci. 2009;122:437-41.

48. Krysko DV, Agostinis P, Krysko O, Garg AD, Bachert C, Lambrecht BN, et al. Emerging role of damage-associated molecular patterns derived from mitochondria in inflammation. Trends Immunol. 2011;32:157-64.

49. Su X, Shen Z, Yang Q, Sui F, Pu J, Ma J, et al. Vitamin C kills thyroid cancer cells through ROS-dependent inhibition of MAPK/ERK and PIBK/AKT pathways via distinct mechanisms. Theranostics. 2019;9:4461-73.

50. Wang C, Li P, Xuan J, Zhu C, Liu J, Shan L, et al. Cholesterol enhances colorectal cancer progression via ROS elevation and MAPK signaling pathway activation. Cell Physiol Biochem. 2017;42:729-42.

51. Zhao Y, Hu X, Liu Y, Dong S, Wen Z, He W, et al. ROS signaling under metabolic stress: cross-talk between AMPK and AKT pathway. Mol Cancer. 2017;16:79.

52. Mi XJ, Hou JG, Wang Z, Han Y, Ren S, Hu JN, et al. The protective effects of maltol on cisplatin-induced nephrotoxicity through the AMPK-mediated PI3K/Akt and p53 signaling pathways. Sci Rep. 2018;8:15922.

53. Hao B, Xiao Y, Song F, Long X, Huang J, Tian M, et al. Metformin-induced activation of AMPK inhibits the proliferation and migration of human aortic smooth muscle cells through upregulation of p53 and IFI16. Int J Mol Med. 2018;41:1365-76.

54. Chen X, Li X, Zhang W, He J, Xu B, Lei B, et al. Activation of AMPK inhibits inflammatory response during hypoxia and reoxygenation through modulating JNK-mediated NF-KB pathway. Metabolism. 2018;83:256-70.

55. Li C, Dong Y, Wang L, Xu G, Yang Q, Tang X, et al. Ginsenoside metabolite compound $\mathrm{K}$ induces apoptosis and autophagy in non-small cell lung cancer cells via AMPK-mTOR and JNK pathways. Biochem Cell Biol. 2019;97:406-14.

56. Luna-Vargas MPA, Chipuk JE. The deadly landscape of pro-apoptotic $\mathrm{BCL}-2$ proteins in the outer mitochondrial membrane. FEBS J. 2016;283:2676-89.

57. Chen $M B$, Zhang $Y$, Wei $M X$, Shen $W, W u X Y$, Yao $C$, et al. Activation of AMP-activated protein kinase (AMPK) mediates plumbagin-induced apoptosis and growth inhibition in cultured human colon cancer cells. Cell Signal. 2013;25:1993-2002.

58. Dann SM, Spehlmann ME, Hammond DC, limura M, Hase K, Choi L, et al. IL-6-dependent mucosal protection prevents establishment of a microbial niche for attaching/effacing lesion-forming enteric bacterial pathogens. J Immunol. 2008;180:6816-26.

59. Deng F, Peng L, Li Z, Tan G, Liang E, Chen S, et al. YAP triggers the Wnt/ $\beta$ catenin signalling pathway and promotes enterocyte self-renewal, regeneration and tumorigenesis after DSS-induced injury. Cell Death Dis. 2018;9:153.

60. Mu Q, Su G, Li L, Gilbertson BO, Yu LH, Zhang Q, et al. Size-dependent cell uptake of protein-coated graphene oxide nanosheets. ACS Appl Mater Interfaces. 2012;4:2259-66.

\section{Publisher's note}

Springer Nature remains neutral with regard to jurisdictional claims in published maps and institutional affiliations. 